\title{
CONDICIONES DE SALUD BUCODENTAL EN NIÑOS MENORES DE CATORCE AÑOS CON LEUCEMIA LINFOBLÁSTICA AGUDA, ANTES DEL TRATAMIENTO DE QUIMIOTERAPIA EN EL INSTITUTO DEL CÁNCER (SOLCA), CUENCA
}

\section{ORO-DENTAL HEALTH CONDITIONS IN CHILDREN UNDER FOURTEEN YEARS WHO SUFFER ACUTE LINPHOBLASTIC LEUKEMIA (ALL) BEFORE RECEIVING TREATMENT AND ASSIST TO THE INSTITUTE OF CANCER IN CUENCA CITY (SOLCA)}

\author{
Janeth Parra Coronel* \\ María Caridad Alvarado Moreno* \\ Pablo Monsalve Toral ${ }^{\cdots *}$ \\ Gonzalo Montesinos Parra***
}

\begin{abstract}
RESUMEN
Los objetivos de esta investigación son: establecer las condiciones de salud bucodental y las características sociodemográficas de los niños con Leucemia Linfoblástica Aguda (LLA) menores de 14 años que acuden al Instituto del Cáncer de Cuenca (SOLCA) y compararlos con los niños sanos que asisten a la clínica de Odontopediatría de la Facultad de Odontología de la Universidad de Cuenca; previo a que los niños reciban sus tratamientos respectivos. Se realiza un estudio analítico transversal en 32 niños con LLA y 32 niños sanos, emparejados por edad y sexo. Las características generales y sociodemográficas de los niños fueron aproximadamente similares; en la talla del rango de edad: $>4$ a 7 años, se encontró que la diferencia estadística $\left(X^{2}\right)$ fue significativa $p<0,03$, la talla fue mayor para el grupo con LLA. En lo que se relaciona a ingesta de medicamentos, exposición a factores ambientales y servicios básicos, las diferencias fueron estadísticamente significativas entre los dos grupos con valores de $p=<0,00 ; p=<0,000 ; p=<0,002$ respectivamente; el grupo con LLA resultó ser más vulnerable que el grupo sano. Al comparar las manifestaciones bucales diagnosticadas entre los dos grupos, las diferencias estadísticas $\left(X^{2}\right)$ fueron significativas en lo que se refiere a equimosis, palidez de las mucosas, sangrado espontáneo de las encías en una o dos piezas dentarias, petequias y ganglios linfáticos palpables, con valores de $p=<0,01 ; p=<0,001 ; p=<0,01 ; p=<0,005 ; p=<0,005$ para cada uno. Los niños con LLA presentaron manifestaciones bucales en mayor porcentaje que los niños sanos. La diferencia de medias (prueba T) del I. Gingival Löe y Silness fue significativamente mayor en el grupo de niños con LLA que en el grupo control, $0,97 \pm 0,56$ vs. $0,43 \pm 0,4, p=<0,00$. Este estudio revela que los niños con LLA constituye una población vulnerable a sufrir lesiones estomatológicas y que es prioritario que la atención odontológica sea incluida en los protocolos de atención del paciente infantil oncológico.
\end{abstract}

Descriptores: Leucemia Linfoblástica Aguda • Salud bucal • Datos demográficos.

\section{ABSTRACT}

The objectives of this research are: to establish the oro-dental health conditions and the socio-demographic features of children who suffer acute linphoblastic leukemia (ALL) who are under fourteen years of age and assist to the institute in cancer of Cuenca city (SOLCA) and to compare them with healthy children who assist to the Odontopediatric Clinic of the Dentistry School of the University of Cuenca; before all children receive their respective treatment. This is an analytical transversal study, which includes 32 children suffering ALL and 32 healthy children; coupled by age and gender. The sociodemographic features of ALL children were approximately the same. The age scale is : $>4-7$ years old; in which we found a significative difference $p<0,03$; being greater for the group. In relation to medicines intake; exposition to environmental factors and basic social services, the differences were statistically significative between the two groups with $p$ valves: $p=<0,00$; and $p<0,02$ for each one. The ALL group is more vulnerable than the healthy group. Comparing the oro-dental lesions between the two groups, the differences were statistically significative $\left(X^{2}\right)$ refering to: ecchymosis, mucose pallor, spontaneous bleeding of gums in one or two teeth units, petechiaes and lymph node enlargement, the following $p$ values: were obtained $p=<0,01 ; p=<0,001 ; p=<0,005$ for each one. The ALL children group showed a larger frequence of oro-dental pathological signs, compared to the healthy children group. The mean

* Especialista en Odontopediatría. Universidad de Buenos Aires, Argentina. Máster en Investigación en Salud - Universidad de Cuenca-Ecuador. Docente de Odontopediatría de la Facultad de Odontología Universidad de Cuenca- Ecuador. Email: judith.parra @ucuenca.edu.ec

** Doctora en Psicología. Universidad Autónoma de Barcelona, España. Psicóloga Clínica Infantil del Instituto del Cáncer, SOLCA-Núcleo de Cuenca. Jefe del Departamento de Apoyo Psicosocial del Instituto del Cáncer, SOLCA-Núcleo de Cuenca. Email: mariacaridad.alvaradomoreno@gmail.com

*** Especialista en Hematología Oncológica Pediátrica. Universidad de Chile. Hematólogo-Oncólogo Pediatra del Instituto del Cáncer, SOLCA-Núcleo de Cuenca. Jefe del Departamento de Pediatría del Instituto del Cáncer, SOLCA-Núcleo de Cuenca. Email: pmonsalvet@gmail.com

**** Odontólogo, Universidad de Cuenca-Ecuador. Candidato a Especialista y Magíster en Radiología. Universidad de São Paulo, Brasil. Universidad UNICID, São Paulo, Brasil. Email: shalo.montesinos@gmail.com 
difference ( $T$ test) of the Loe y Silness Gingival Index was significantly higher in the ALL group than in the control group: $0,970,56$ vs. $0,430,4 ; p=<0,00$. This research proves that children who suffer ALL, are vulnerable to present estomatological lesions; and therefore it becomes a priority to include dental care in the guideliness of medical care for children who suffer oncological conditions.

Descriptors: Acute Linphoblastic leukemia • Oral health • Demographic data.

\section{INTRODUCC I ÓN}

La palabra leucemia designa a un grupo de neoplasias, biológicamente heterogéneas, generadas por alteraciones de los circuitos genéticos que regulan la vida, crecimiento, diferenciación y muerte de las células progenitoras hematopoyéticas $^{1}$. El clon leucémico aumenta progresivamente en número y suprime el crecimiento de las células hematopoyéticas normales ${ }^{2}$.

La Leucemia Linfoblástica Aguda (LLA) representa aproximadamente $25 \%$ de los diagnósticos de cáncer en los niños menores de 15 años, siendo más común en niños hispanos ${ }^{3}$. La LLA en el Instituto del Cáncer SOLCA, Cuenca-Ecuador en el año 2008 ocupó el primer lugar dentro de las neoplasias más frecuentes en niños menores de 14 años, el cuarto lugar de mortalidad en varones y el sexto en mujeres $^{4}$. Los niños con LLA presentan manifestaciones bucales que son propias de la enfermedad. Según el Instituto Nacional del Cáncer, antes del tratamiento oncológico, se deben tratar problemas orales que ya existen, para prevenir complicaciones orales e impedir dificultades durante $y$ después del tratamiento ${ }^{5}$.

Los signos y síntomas de la LLA en niños pueden ser vagos, incluyendo fiebre persistente, fatiga, hematomas, petequias, sangrado de las mucosas, dolor de huesos y linfadenopatía, entre otros ${ }^{6}$. Los primeros signos de leucemia, especialmente en la fase aguda, pueden manifestarse en la cavidad oral debido a la trombocitopenia, neutropenia, o a la función comprometida de los granulocitos; estas lesiones pueden ser evaluadas y diagnosticadas por el Odontopediatra, muchas veces es el primer profesional de la salud que descubre la leucemia aguda en niños ${ }^{7}$. La relación entre la frecuencia de las manifestaciones orales y la leucemia antes del tratamiento ha sido evidenciada en pocos estudios, el sangrado oral y petequias fueron los signos orales más comunes de la misma ${ }^{8}$, Orback R y Orback Z $Z^{9}$ (1997) reportaron que en 47 pacientes que padecían de LLA, el $68,1 \%$ presentaban palidez de la mucosa oral, $14,9 \%$ petequias, $6,4 \%$ sangrado gingival espontáneo, $14,9 \%$ herpes labial, 23,4\% candidiasis oral, existiendo hallazgos muy similares en otro estudio ${ }^{10}$. Revisiones sistemáticas han reportado que las inflamaciones periodontales, gingivitis y sangrado de la encías son significativamente mayores en los niños con LLA en comparación con los niños controles sanos $^{11,12}$. En síntesis las manifestaciones más frecuentes al examen odontológico son: Linfoadenopatia, palidez de la mucosa oral, sangrado de las encías, petequias, úlceras, lesiones herpéticas, gingivitis ${ }^{13,14}$ por lo cual el mantenimiento de una buena higiene oral en estos niños hospitalizados es prioritario ${ }^{15,16,17}$.

La LLA está relacionada también con las características sociodemográficas de la familia que indican que el ambiente cotidiano del niño puede influir en la misma ${ }^{18}$ 19, 20, 21 , a su vez influyen en la salud bucodental tanto en niños sanos como con $\operatorname{LLA}^{22}$.

Este estudio tiene por objeto determinar las condiciones de salud bucodental y sociodemográficas de los niños con LLA menores de 14 años y compararlos con los de los niños sanos.

\section{MATERIALES Y MÉTODOS}

Se trata de un estudio analítico de corte transversal, que se desarrolló en el periodo 2012 - 2015, en niños menores de 14 años con LLA y niños controles (sanos) que acudieron por primera vez a SOLCA y a la Facultad de Odontología, para recibir los tratamientos respectivos.

La tasa de incidencia de LLA en niños menores de 14 años en SOLCA fue de 9,02 por cien mil niños (año 2005); de
PARRA J C
ALVARADO MCM
MONSALVE PT
MONTESINOS GP
CONDICIONES
DE SALUD
BUCODENTAL EN
NIÑOS MENORES
DE CATORCE AÑOS
CON LEUCEMIA
LINFOBLÁSTICA
AGUDA,
ANTES DEL
TRATAMIENTO DE
QUIMIOTERAPIA
EN EL INSTITUTO
DEL CANCER
(SOLCA), CUENCA

195

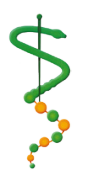

REV, ODONTOL.

UNIV, CID, SÃO

PAULO

2016; 28(3):

$194-203$, SET - DEZ 
PARRA JC

ALVARADO MCM

MONSALVE PT

MONTESINOS GP

CONDICIONES

$D E \quad S A L U D$

BUCODENTAL EN

NIÑOS MENORES

DE CATORCE AÑOS

CON LEUCEMIA

LINFOBLÁSTICA

$A G \cup D A$,

ANTES DEL

TRATAMIENTO DE

QUIMIOTERAPIA

EN EL INSTITUTO

DEL CÁNCER

(SOLCA), CUENCA

\section{$196 \cdots$}

REV, ODONTOL.

UNIV, CID, SÃO

PAULO

2016; 28(3):

194-203, SET - DEZ acuerdo a los datos del INEC en Cuenca en el año 2005 hubieron 140.000 niños, por lo tanto se infiere que la incidencia de niños con LLA en el cantón Cuenca es de trece casos por año, pudiendo suceder que algunos padres de los niños no firmen el consentimiento, por lo cual se estudiaron a 64 niños en tres años, 32 niños con LLA y 32 niños sanos (controles).

Los niños menores de 14 años se incluyeron de forma consecutiva no aleatoria (emparejados por edad, sexo), que tengan el consentimiento firmado por sus padres o representantes. Se excluyeron los niños que estén en tratamiento de la LLA en cualquiera de sus fases, que presenten otras enfermedades como alteraciones cromosómicas, parálisis cerebral, cardiopatías congénitas entre otras y niños que hayan recibido tratamiento con quimioterapia; en los niños controles con consentimiento informado firmado, los criterios de exclusión comprendieron a niños que sufran de enfermedades crónicas o con discapacidades, niños con uso crónico de medicamentos y que estén bajo tratamiento odontológico. El estudio fue aprobado por el Comité de Ética (Memorando No. 318-FAO-2011 de la Facultad de Odontología de la Universidad de Cuenca (Cuenca, Ecuador).

Recolección de datos generales y sociodemográficos de los niños

Se aplicó encuestas estandarizadas a los padres/representantes, que contenían preguntas sobre datos de filiación del niño, antecedentes médicos y datos sociodemográficos.

El día en que el niño ingresó al hospital, la enfermera registró la talla y el peso de cada uno de forma estandarizada (balanza y tallímetro calibrados), aparatos de marca "health o meter". El registro de los niños controles se efectuó con estos mismos procedimientos.

\section{Evaluación Oncológica}

Todos los exámenes de los niños con LLA se ejecutaron siguiendo el protocolo de atención de SOLCA. El Oncólogo Pediatra diagnosticó las lesiones bucales oncológicas de los niños con LLA; la exploración se lo realizó con ayuda de un baja lenguas y una linterna. Se examinó si el niño presentaba: decoloraciones blan- quecinas; candidiasis; úlceras; eritema, herpes labial; área de necrosis; dificultad para deglutir sólidos, líquidos y dificultad para hablar. Sapp J.F F $^{14}$ (2004).

\section{Evaluación clínica odontológica}

Para efectuar este examen previamente se coordinaba con el médico oncólogo. El examen extrabucal de la cabeza, cara y cuello se valoró mediante la inspección y palpación; se buscó nódulos linfáticos palpables y se examinó la piel perioral. Para realizar examen intrabucal, el niño efectuaba un enjuague bucal con clorhexidina al $0,12 \%^{23}$ y para la valoración gingival se complementaba con una nueva aplicación a nivel local, se buscaron lesiones leucémicas como: palidez de la mucosa bucal; petequias; sangrado de las encías; equimosis; púrpura e hiperplasia gingival. Ceccottis y Sforza ${ }^{24}$ (2007).

La valoración gingival de los niños se hizo con el Índice Gingival Löe H. y Silness $^{25}$ (1963), utilizando un espejo bucal plano y una sonda periodontal de la OMS (CP11), marca Hu-friedy. A los niños que presentaban la encía eritematosa y sangrante se les atribuía el valor 3 , sin necesidad del examen correspondiente.

Terminado el registro de datos, se orientó a los padres sobre la higiene oral de los niños y se les aplicó medidas preventivas, basados en los criterios recomendados por la Academia Dental Americana de Odontopediatría ${ }^{26}$. A los niños control se les hizo todos los exámenes y procedimientos anteriores.

Los datos se recogieron en el formulario diseñado y luego de revisados se transfirieron a una base de datos del programa SPSS v. 22. Se calculó la frecuencia de las variables cualitativas; además de algunas variables cuantitativas se obtuvieron promedios y desvió estándar (SD). Las diferencias de proporciones se examinaron mediante la prueba de Chi cuadrado de Pearson y la diferencia de promedios mediante la prueba t Student para muestras independientes. Se compararon las lesiones bucales entre los niños con LLA y los niños sanos mediante tablas de contingencia dos por dos y pruebas de Chi cuadrado, o prueba de Fisher cuando el número de observaciones fue menor a 5 , con una con un nivel de significancia del 0,05. 
Tabla 1. Comparaciones basales de las características generales entre los niños con leucemia linfoblástica aguda y niños sanos.

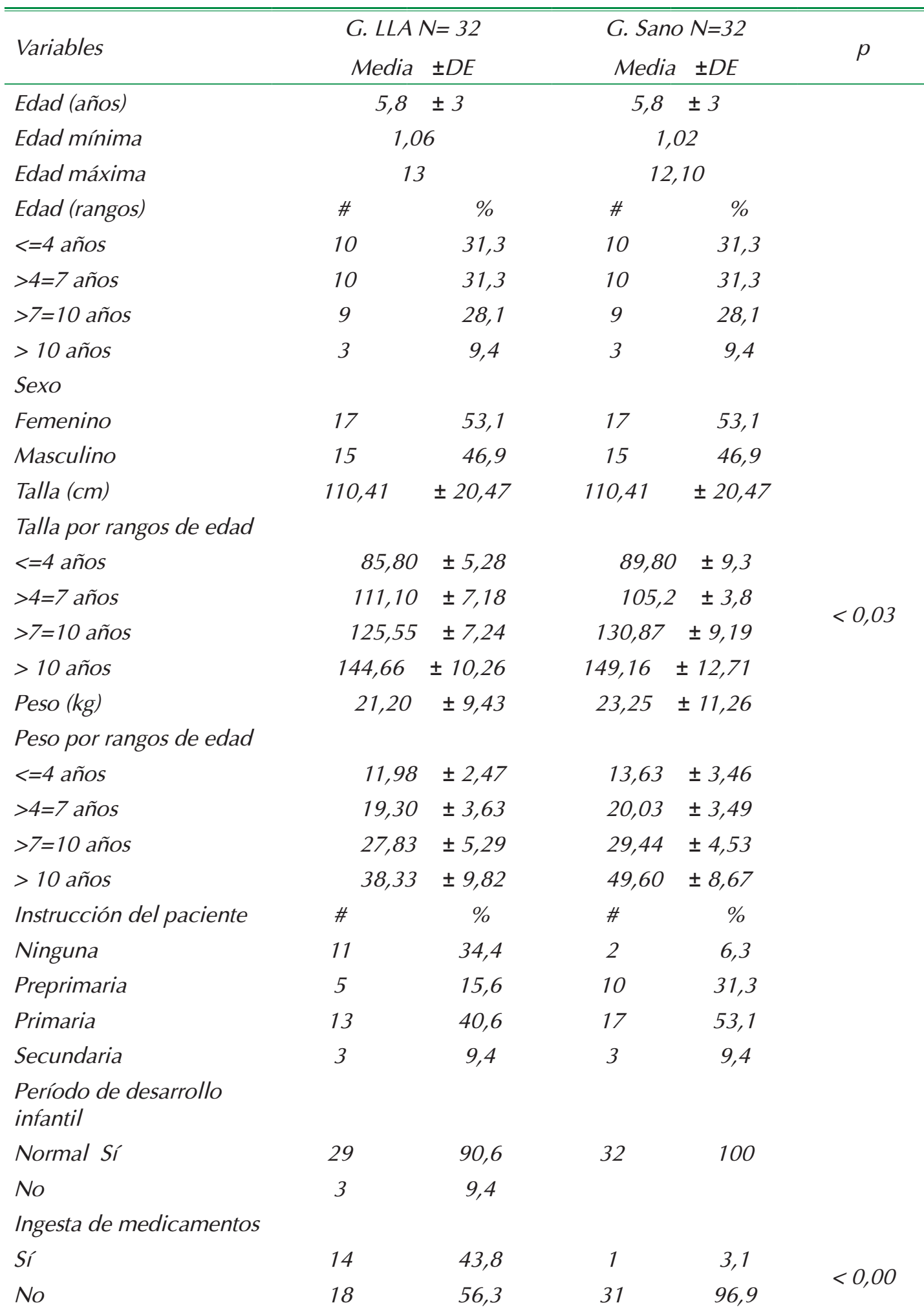

\section{RESULTADOS}

Las características generales de los dos grupos de estudio se observan en la Tabla1.

La edad promedio de los niños fue de 5 años 8 meses ( \pm 3 meses), la talla, peso,

talla y peso por rango de edad de los dos grupos fueron semejantes, a excepción de la talla del rango de edad $>4=7$ años, en el cual se encontró que la diferencia estadística $\left(\mathrm{X}^{2}\right)$ fue significativa, la talla fue mayor para el grupo con LLA. En cuanto a los niveles de escolaridad y desarrollo infantil,
PARRA J $C$ ALVARADO MCM MONSALVE PT MONTESINOS GP

CONDICIONES DE SALUD BUCODENTAL EN NIÑOS MENORES DE CATORCE AÑOS CON LEUCEMIA

LINFOBLÁSTICA $A G \cup D A$,

ANTES DEL tRATAMIENTO DE QUIMIOTERAPIA en el INSTITUTO DEL CÁNCER (SOLCA), CUENCA 
PARRA JC ALVARADO MCM MONSALVE PT MONTESINOS GP

CONDICIONES DE SALUD BUCODENTAL EN NIÑOS MENORES DE CATORCE AÑOS CON LEUCEMIA

LINFOBLÁSTICA $A G \cup D A$, ANTES DEL TRATAMIENTO DE QUIMIOTERAPIA EN EL INSTITUTO DEL CÁNCER (SOLCA), CUENCA

REV. ODONTOL UNIV, CID, SÃO PAULO $2016 ; 28(3):$ $194-203$, SET - DEZ

Tabla 2. Características sociodemográficas de los niños con leucemia linfoblástica aguda y niños sanos.

\begin{tabular}{llllll}
\hline \hline \multirow{2}{*}{ Variables } & G. caso & \multicolumn{3}{l}{ G. control } \\
& $\mathrm{N}=32$ & \multicolumn{5}{c}{$\mathrm{N}=32$} & & \\
\hline Sector & $\#$ & $\%$ & $\#$ & $\%$ & $\mathrm{p}$ \\
Urbano & 18 & 56,3 & 30 & 93,8 & \\
Rural & 14 & 43,8 & 2 & 6,3 &
\end{tabular}

Tipo de familia

Nuclear

Padres separados

Madre soltera

Mono parenteral

Extensa-consanguínea

$10 \quad 31,3 \quad 20 \quad 62,5$

Tipo de vivienda

Casa

Departamento

$\begin{array}{llll}3 & 9,4 & 9 & 28,1\end{array}$

$\begin{array}{llll}1 & 3,1 & 1 & 3,1\end{array}$

$\begin{array}{llll}2 & 6,3 & 2 & 6,3\end{array}$

Media agua

16

50,0

0,0

2 - 3 cuartos

1 cuarto

$\begin{array}{llll}18 & 56,3 & 21 & 67,7 \\ 9 & 28,1 & 8 & 22,6\end{array}$

67,7

$\begin{array}{llll}2 & 6,3 & 1 & 3,2\end{array}$

$\begin{array}{llll}1 & 3,1 & 0 & 0,0\end{array}$

Agua de consumo

Potable

6,3

6,5

Entubada

No entubada - vertiente

2

2

3,2

0

24,0

Pozo

No potable: tanquero

$\begin{array}{llll}24 & 75,0 & 30 & 93,8\end{array}$

$\begin{array}{llll}3 & 9,4 & 2 & 6,3\end{array}$

$2 \quad 6,3 \quad 0 \quad 0,0$

$2 \quad 6,3 \quad 0 \quad 0,0$

Baño

Sí

1

3,1

0,0

No

2
8

Expuesto a factores ambientales

Sí

No

Ingreso mensual familiar

los resultados son aproximados; 14 niños $(43,8 \%)$ con LLA tienen antecedentes de ingesta de medicamentos (desde 2 meses atrás) y 1 niño (3,1\%), del grupo control, la diferencia estadística fue significativa $\left(X^{2}\right)$, el grupo de niños con LLA tiene más antecedentes de ingesta de medicamentos.

Las características del medio familiar de los dos grupos de estudio se observan en la Tabla 2.

En los niños con LLA, 16 (50\%) pertenecen a familias extensas-consanguíneas, los niños control no tienen este tipo de familia, en este grupo el mayor porcentaje

$\begin{array}{llll}75,0 & 32 & 100 & <0,002 \\ 25,0 & 0 & 0 & \end{array}$

$\begin{array}{clcl}59,4 & 1 & 3,1 & <0,000 \\ 40,6 & 31 & 96,9 \\ & \$ 597,42 & \end{array}$

de niños tiene familia nuclear $20(62,5 \%)$. En cuanto al tipo de vivienda los datos son similares en los dos grupos; la mayoría de los niños consumen agua potable, sin embargo en el grupo de niños con LLA existe un número menor que consume agua de vertiente, pozo y tanquero. Todos los niños del grupo control tienen servicios básicos vs. el 75\% del grupo con LLA; la diferencia fue estadísticamente significativa. Los niños con LLA estuvieron más expuestos a factores ambientales que los control $(59,4 \%)$ vs. $(3,1 \%)$; la diferencia estadística fue significativa. El ingreso mensual familiar de los dos grupos es 
aproximadamente equivalente.

Las manifestaciones bucales que se diagnosticaron en los niños con LLA y los niños sanos antes del tratamiento se observan en la Tabla 3.

Las manifestaciones fueron significativamente mayores en niños con LLA; a excepción de la presencia de úlceras que no hubo diferencia entre los grupos, además en un niño con LLA se encontró sangrado espontáneo de todas sus encías. Las lesiones se ubicaron en los labios, mucosa yugal, paladar, piso de la boca, lengua y encías.

La evaluación del estado gingival de los niños con leucemia linfoblástica aguda y niños sanos se observan en la Tabla 4. La G. incipiente es la más frecuente en los dos grupos $23(71,9 \%)$ vs. 24 (75\%), la moderada es más frecuente en los niños con LLA que en los controles, 8 (25\%) vs.2 (6,3\%), un niño con LLA presentó G. severa; en el grupo de niños control no se observó G. severa y hubieron 6 niños $(18,7 \%)$ sanos.

La evaluación de la media y DS del I. Gingival Löe y Silness ${ }^{25}$, de los dos grupos de niños se observan en la Tabla 5.

La media del IGLS para el grupo con LLA fue de $0,97 \pm 0,56$ y en el grupo control de $0,43 \pm 0,41$; la prueba $T$ para muestras independientes fue estadísticamente significativa, la media de los niños con LLA es mayor que en el grupo control.

\section{I SCUSIÓN}

Actualmente se ha determinado que a más de los factores biológicos que intervienen en la LLA, la caracterización de filiación y sociodemográfica de los niños con LLA son variables importantes que pueden influenciar en la misma y tienen impacto en la salud bucodental tanto en niños con LLA como en los niños sanos $18,22,26$. Se reportaron diferencias estadísticas significativas en cuanto a la ingesta de

Tabla 3. Manifestaciones bucales de los niños con leucemia linfoblástica aguda y niños sanos.

\begin{tabular}{lccccc}
\hline \hline & \multicolumn{4}{c}{ Niños LLA } & \multicolumn{2}{c}{ Niños sanos } & p \\
& \multicolumn{2}{c}{$\mathrm{N}=32$} & \multicolumn{2}{c}{$\mathrm{N}=32$} & \\
\hline Manifestaciones clínicas & $\#$ & $\%$ & $\#$ & $\%$ & \\
Equimosis: sí & 8 & 25,0 & 1 & 3,1 & $<0,01$ \\
Úlceras: sí & 3 & 9,4 & 2 & 6,3 & \\
Palidez de la mucosa oral: sí & 10 & 31,3 & 0 & 0,0 & $<0,001$ \\
Sangrado espontáneo en 1-2 & 12 & 37,5 & 3 & 9,3 & $<0,01$ \\
piezas: sí & & & & & \\
Sangrado espontáneo genera- & 1 & 3,5 & 0 & 0,0 & \\
lizado encías: sí & & & & & \\
Petequias: sí & 15 & 46,9 & 4 & 12,5 & $<0,005$ \\
G. linfáticos palpables: sí & 20 & 62,4 & 8 & 25,0 & $<0,005$ \\
\hline \hline
\end{tabular}

Test chi-cuadrado

Tabla 4. Índice gingival de Löe y Silness de los niños con leucemia linfoblástica aguda y niños sanos.

\begin{tabular}{lcccc}
\hline \hline & \multicolumn{2}{c}{ Niños LLA } & \multicolumn{2}{c}{ Niños sanos } \\
& \multicolumn{2}{c}{$\mathrm{N}=32$} & & \multicolumn{2}{c}{$\mathrm{N}=32$} \\
\hline Índice Gingival Löe y Silness & $\#$ & $\%$ & & $\%$ \\
Normal & 0 & 0,0 & 6 & 18,7 \\
Gingival incipiente & 23 & 71,9 & 24 & 75,0 \\
Gingival moderada & 8 & 25,0 & 2 & 6,3 \\
Gingival severa & 1 & 3,1 & 0 & 0,0 \\
Total & 32 & 100,0 & 32 & 100,0 \\
\hline \hline
\end{tabular}

PARRA J C ALVARADO MCM MONSALVE PT MONTESINOS GP

CONDICIONES DE SALUD BUCODENTAL EN NIÑOS MENORES DE CATORCE AÑOS CON LEUCEMIA

LINFOBLÁSTICA $A G \cup D A$,

ANTES DEL TRATAMIENTO DE QUIMIOTERAPIA EN EL INSTITUTO DEL CÁNCER (SOLCA), CUENCA

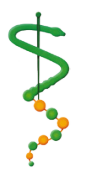

REV, ODONTOL. UnIV. CID. São PAULO

2016; 28(3):

194-203,SET-DEZ 
PARRA JC

ALVARADO MCM

MONSALVE PT

MONTESINOS GP

CONDICIONES

$D E \quad S A L U D$

BUCODENTAL EN

NIÑOS MENORES

DE CATORCE AÑOS

CON LEUCEMIA

LINFOBLÁSTICA

$A G \cup D A$,

ANTES DEL

TRATAMIENTO DE

QUIMIOTERAPIA

EN EL INSTITUTO

DEL CÁNCER

(SOLCA), CUENCA

\section{$200 \ldots$}

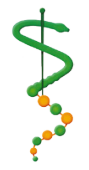

REV, ODONTOL.

UNIV, CID, SÃO

PAULO

$2016 ; 28(3):$

$194-203$, SET - DEZ
Tabla 5 Comparación del promedio del índice gingival de Löe y Silness, entre los niños con leucemia linfoblástica aguda y niños sanos

\begin{tabular}{lccc}
\hline \hline & Niños LLA & Niños sanos & \\
Índice Gingival Löe y Silness & Media \pm DE & Media \pm DE & $P$ \\
& $0,97 \pm 0,56$ & $0,43 \pm 0,41$ & $<0,00$ \\
\hline \hline
\end{tabular}

Prueba T muestras independientes

medicamentos, exposición a factores ambientales y servicios básicos, que indican que los niños con LLA son más vulnerables que los niños controles; nuestro hallazgo indica que el 59\% de los niños con LLA estuvieron o están expuestos durante su primera infancia a factores ambientales (fungicidas/sustancias de latonería). En la encuesta ejecutada no se consideró si esta exposición la tuvo también la madre durante el embarazo, dato importante, ya que según algunos estudios exponen que diferentes exposiciones ambientales prenatales pueden constituir factores de riesgo de la LLA $20,21,27$

La mayoría de niños de los dos grupos consumen agua potable, sin embargo, en el grupo con LLA, un porcentaje menor consume agua no entubada - vertiente, pozo y no potable-tanquero; además un $25 \%$ de los niños con LLA no tienen servicios básicos, lo que indica que los niños con LLA están más afectados.

Al comparar las condiciones de salud bucodental entre los dos grupos, las diferencias fueron estadísticamente significativas en equimosis, palidez de la mucosa oral, sangrado espontáneo de las encías en una o dos piezas, petequias y ganglios linfáticos palpables, los niños con LLA presentaron lesiones estomatológicas patológicas en mayor porcentaje que los niños sanos. En un estudio realizado por Orback R y Orback Z9 (1997), las lesiones más frecuentes en niños con LLA fueron: palidez de la mucosa oral $(68 \%)$, resultado que es más del doble en relación a nuestro resultado $(31,3 \%)$; petequias $(14,9 \%)$, frecuencia más baja en contraste a nuestro reporte $(46,9 \%)$ y sangrado espontáneo de las encías $(6,4 \%)$, el doble al encontrado en nuestro estudio $(3,5 \%)$. Hou et al. ${ }^{28}$ (1997) reportaron linfoadenopatía en el $(71,4 \%)$ de los niños, superior al de nuestro estudio $(62,4 \%)$ y sangrado de las encías en el $(28,6 \%)$, en este estudio no se especifica si es generalizado o parcial; en nuestro resultado solamente un niño con LLA presentó sangrado de todas las encías $(3,5 \%)$ y sangrado a nivel de una o dos piezas dentarias $(37,5 \%)$; esta discrepancia pueda deberse a la diferencia de tamaño de las muestras. Los hallazgos concuerdan con revisiones científicas sobre los signos y síntomas bucales de la LLA antes del tratamiento con quimioterapia ${ }^{17,29}$ Según Wecki et al. ${ }^{10}$ (1990), Freitas y Consolaro ${ }^{11}$ (1990), las manifestaciones de sangrado gingival bien pueden ser el resultado de la infiltración de células leucémicas (primaria) o (secundaria) trombocitopenia subyacente, neutropenia, o alteración función de los granulocitos. El sangrado gingival se puede presentar en ausencia de gingivitis detectable clínicamente y puede ser un signo precoz de la enfermedad ${ }^{30}$. Sería fundamental realizar nuevos estudios sobre la salud periodontal y su relación con factores hematológicos.

En el presente estudio, tanto los niños del grupo LLA y control presentaron mayor frecuencia de Gingivitis incipiente; la gingivitis moderada fue más frecuente en el grupo con LLA y la gingivitis severa solo se presentó en un niño con LLA. Seis niños controles no presentaron gingivitis. tudio la G. moderada (25\%) y la G. severa $(3,1 \%)$ en los niños con LLA, fueron menos frecuentes en relación a un estudio similar en el cual el grupo de niños con LLA tuvieron un $(80 \%)$ de G. moderada y un (13\%) G. severa; no se presentó G. severa en los niños control de los dos estudios ${ }^{31}$. La media de la gingivitis fue significativamente mayor en el grupo de niños con LLA que en el grupo control, 0,97 vs. 0,43 respectivamente, resultado superior a la media de 0,084 en niños con LLA y de 0,003 en los niños control, reportado por Pels y Mielnik-Błaszczak ${ }^{32}$ (2012), pero inferior en relación a otros estudios en los que se encontraron promedios de 1,5 y de 1,20 en niños con $\operatorname{LLA}^{31,33}$, Al comparar los resultados de nuestro es- 
resultados que coinciden con los obtenidos por Javed et al. ${ }^{12}$ (2012), que encontraron que la gingivitis es común en niños con LLA en relación al grupo control; en nuestro estudio el grado 1 de gingivitis fue el más frecuente.

\section{CONCLUSIONES Y RECOMENDACIONES}

Las características generales y sociodemográficas fueron aproximadamente similares en los dos grupos de niños, sin embargo en cuanto a la ingesta de medicamentos, servicios básicos y exposición a factores ambientales, los niños con LLA son más vulnerables que los niños control.

La salud oral, antes de recibir el respectivo tratamiento en los niños con LLA, está más comprometida que los control. El sangrado gingival espontáneo ya sea parcial - total en los niños con LLA estuvo relacionado posiblemente con la trombocitopenia que presentaron los mismos. La aplicación de medidas de prevención bucodental ayudó a que no se presenten complicaciones de las lesiones encontradas, por lo tanto es prioritario que se in- corpore al Odontólogo en el equipo multidisciplinario de salud de los hospitales oncológicos.

Se recomienda realizar estudios de los factores de riesgo asociados a la LLA de acuerdo a nuestras características poblacionales.

\section{AGRADEC IMIENTOS}

Agradecemos a la Dirección de Investigación de la Universidad de Cuenca (DIUC), por el financiamiento otorgado para el desarrollo de esta investigación; a SOLCA - (Cuenca) y su personal, al Dr. Raúl Alvarado C. Director del Instituto, por el apoyo financiero y logístico para realizar el estudio; al equipo de investigadores, pero sobre todo a los niños y padres de familia por la colaboración brindada en este estudio.

Un agradecimiento especial a los estudiantes ayudantes de Investigación: Andrea Flores Flores; Rubén León Vásquez; Marcos Mendoza Yamunaque; Cristian Paredes Pacheco; Verónica Rivas Barrionuevo.
PARRA J C ALVARADO MCM MonsalVE PT MONTESINOS GP

CONDICIONES DE SALUD BUCODENTAL EN NIÑOS MENORES DE CATORCE AÑOS CON LEUCEMIA LINFOBLÁSTICA $A G \cup D A$,

ANTES DEL TRATAMIENTO DE QUIMIOTERAPIA EN EL INSTITUTO DEL CÁNCER (SOLCA), CUENCA

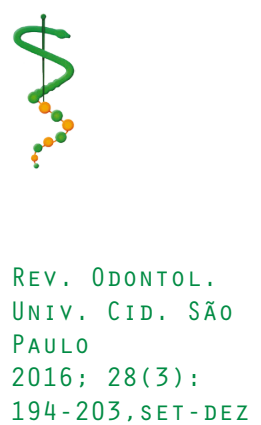


PARRA JC ALVARADO MCM

MONSALVE PT

MONTESINOS GP

CONDICIONES

$D E \quad S A L U D$

BUCODENTAL EN

NIÑOS MENORES

DE CATORCE AÑOS

CON LEUCEMIA

LINFOBLÁSTICA

$A G \cup D A$,

ANTES DEL

TRATAMIENTO DE

QUIMIOTERAPIA

EN EL INSTITUTO

DEL CÁNCER

(SOLCA), CUENCA

202
1. Greaves MF. The new biology of leukemia. In: Henderson, ES, Lister, TA, Greaves, MF. Leukemia. 6. ed. Philadelphia: WB Saunders; 1996. p. 3445.

2. Pui CH. Leucemias infantiles. In: Murphy, GP, Laurence Jr, W, Lenhard Jr, RE. Oncología clínica: manual de la American cancer society. 2. ed. Washington: Organización Panamericana de la Salud; 1996. p. 561-86.

3. Howlader N, Noone AM, Krapcho M. Childhood cancer: section 28. Bethesda: National Cancer Institute; 2013 [Last accessed August 19, 2016]; Disponível em: https://seer.cancer.gov/archive/csr/1975_2010/results_merged/ sect_28_childhood_cancer.pdf.

4. Instituto Del Cáncer Solca Cuenca. Registro de tumores: quinto informe. 2008 [Last accessed August 19, 2016]; Disponible en: http://www.institutodelcancer.med.ec/.

5. Instituto Nacional Del Cáncer. Complicaciones orales de la quimioterapia y la radioterapia a la cabeza y cuello. 2016 [Last accessed Septiembre 2015]; Disponible en: https:// www.cancer.gov/espanol/cancer/tratamiento/efectos-secundarios/boca-garganta/complicaciones-orales-pro-pdq\#section/_16.

6. Howard S. Initial management of the child with suspected leukemia or lymphoma Cure4Kids; 2010 [Last accessed August 5, 2016]; Disponible en: https://www.cure4kids.org/.

7. Deliverska EG, Krasteva A. Oral signs of leukemia and dental managementliterature data and case report. J IMAB 2013 19(4):388-91.

8. Lynch MA, Ship, II. Oral manifestations of leukemia: a postdiagnostic study. Journal of the American Dental Association (1939) 1967 Nov;75(5):113944.
9. Orbak R, Orbak Z. Oral condition of patients with leukemia and lymphoma. The Journal of Nihon University School of Dentistry 1997 Jun;39(2):6770 .

10. Weckx LL, Hidal LB, Marcucci G. Oral manifestations of leukemia. Ear, nose, \& throat journal 1990 May;69(5):3412, 5-6.

11. Freitas TC, Consolaro A. Manifestações bucais das leucemias agudas. Rev odontol Univ São Paulo 1990 jul-set;4(3):261-4.

12. Javed F, Utreja A, Bello Correa FO, Al-Askar M, Hudieb M, Qayyum F, et al. Oral health status in children with acute lymphoblastic leukemia. Critical reviews in oncology/hematology 2012 Sep;83(3):303-9.

13. Fayle SA, Curzon ME. Oral complications in pediatric oncology patients. Pediatric dentistry 1991 Sep-Oct;13(5):289-95.

14. Sapp JP, Eversole LR, Wysocki GP. Patología oral y maxilofacial contemporánea. Madrid España: Elsevier; 2004.

15. Azher U, Shiggaon N. Oral health status of children with acute lymphoblastic leukemia undergoing chemotherapy. Indian journal of dental research : official publication of Indian Society for Dental Research 2013 Jul-Aug;24(4):523.

16. Padmini C, Bai KY. Oral and dental considerations in pediatric leukemic patient. ISRN hematology 2014 $2014(895721$.

17. Zimmermann C, Meurer MI, Grando LJ, Gonzaga Del Moral JA, Da Silva Rath IB, Schaefer Tavares S. Dental treatment in patients with leukemia. Journal of oncology 20152015 (571739.
REV, ODONTOL. UNIV, CID . SÃO: PAULO 2016; 28(3): $194-203$, SET - DEZ 
18. Viana MB, Fernandes RA, De Carvalho RI, Murao M. Low socioeconomic status is a strong independent predictor of relapse in childhood acute lymphoblastic leukemia. International journal of cancer Supplement = Journal international du cancer Supplement 1998 11(56-61.

19. Ribeiro KB, Buffler PA, Metayer C. Socioeconomic status and childhood acute lymphocytic leukemia incidence in Sao Paulo, Brazil. International journal of cancer 2008 Oct 15;123(8):1907-12.

20. Castro-Jimenez MA, Orozco-Vargas LC. Parental exposure to carcinogens and risk for childhood acute lymphoblastic leukemia, Colombia, 20002005. Preventing chronic disease 2011 Sep;8(5):A106.

21. Pérez-Saldivar ML, Mejía-Aranguré J, Rangel-López A, Fajardo Gutiérrez A. Sociodemographic and birth characteristics in infant acute leukemia: a review. Clinic Epidemiol Acute Lympho Leuk From Molec Clinic 2013 145-70.

22. Garibotti G, Vasconi C, Ferrari A, Giannini G, Comar H, Schnaiderman D. Parental perception of psychophysical health, nutritional status and oral health in relation to sociodemographic characteristics in children in Bariloche, Argentina: an epidemiological study. Archivos argentinos de pediatria 2015 Oct;113(5):411-8.

23. Cheng KK. Oral mucositis, dysfunction, and distress in patients undergoing cancer therapy. Journal of clinical nursing 2007 Nov;16(11):2114-21.

24. Ceccottis L, Sforza REl. Diagnóstico en clínica estomatológica. 2. ed. Buenos Aires: Editorial Médica Panamericana; 2007.

25. Loe H, Silness J. Periodontal disease in pregnancy. I. Prevalence and severity. Acta odontologica Scandinavica 1963 Dec;21(533-51.
26. Guideline on dental management of pediatric patients receiving chemotherapy, hematopoietic cell transplantation, and/or radiation. Pediatric dentistry 2013 Sep-Oct;35(5):E185-93.

27. Ferreira JD, Couto AC, Pombo-De-Oliveira MS, Koifman S. In utero pesticide exposure and leukemia in Brazilian children $<2$ years of age. Environmental health perspectives 2013 Feb;121(2):269-75.

28. Hou GL, Huang JS, Tsai CC. Analysis of oral manifestations of leukemia: a retrospective study. Oral diseases 1997 Mar;3(1):31-8.

29. Ivanovic M, Jovcic O, Mandic J, Bogetic D, Maddalone M. [Oral manifestations of acute leukaemia]. Srpski arhiv za celokupno lekarstvo 2011 Jan-Feb;139(1-2):103-6.

30. Stafford R, Sonis S, Lockhart P, Sonis A. Oral pathoses as diagnostic indicators in leukemia. Oral surgery, oral medicine, and oral pathology 1980 Aug;50(2):134-9.

31. Venkatesh B, Kavyashree BS. Comparative evaluation of oral health status in children with acute lymphoblastic leukemia. Inter J Scientific Study 2015 02(10):10.

32. Pels E, Mielnik-Blaszczak M. Oral hygiene in children suffering from acute lymphoblastic leukemia living in rural and urban regions. Annals of agricultural and environmental medicine : AAEM 2012 19(3):529-33.

33. Viera NT, Rojas De Morales T, Navas RM, Zambrano OR, Paz De Gudiño M. Gingivitis y anticuerpos anticitoplasmáticos de neutrófilos en niños y adolescentes con leucemia. Med oral patol oral cir bucal 2004 9(5):396402.

Recebido em 18/11/2016

Aceito em 01/12/2016
PARRA J C ALVARADO MCM MONSALVE PT MONTESINOS GP

CONDICIONES DE SALUD BUCODENTAL EN NIÑOS MENORES DE CATORCE AÑOS CON LEUCEMIA LINFOBLÁSTICA $A G U D A$,

ANTES DEL TRATAMIENTO DE QUIMIOTERAPIA EN EL INSTITUTO DEL CÁNCER (SOLCA), CUENCA

\section{3}

EV. ODONTOL.

UNIV, CID, São

PAULO

2016; 28(3):

194-203, SET-DEZ 